\title{
SIQR dynamics in a random network with heterogeneous connections with infection age
}

\author{
Hairong Yan, Jinxian Li \\ School of Mathematical Sciences, Shanxi University, Taiyuan, 030006, P.R. China.
}

\begin{abstract}
In this paper, an SIQR-Epidemic transmission model of the non-Markovian infection process and quarantine process in a heterogeneous complex network is established, in which the infection rate and quarantine rate are related to infection age. Next, we use the method of characteristics to transform the model into an integro-differential equation and derive the epidemic threshold of the model. Finally, we focus on the impact of three different infection or quarantine time distributions on the disease transmission and show that infection or quarantine time distribution has a significant effect on the disease dynamics.
\end{abstract}

Keywords: SIQR-epidemic, complex network, infection age, non-Markovian transmission and quarantine, epidemic threshold, distribution.

2020 MSC: 05C82, 37N25, 92D25.

(C)2021 All rights reserved.

\section{Introduction}

Mathematical model plays an important role in understanding the spread of infectious diseases. It is mainly used to study the spread and control of infectious diseases [11]. Classic infectious disease models of Susceptible-Exposed-Infectious-Recovered (SEIR) were proposed as early as the 20th century [1], and became the basic model of studies on the numerous infectious diseases spreading. Where Exposed (E) stands for latent period, a period in which a susceptible shows no symptoms after infection.

Currently, many epidemic models with latent periods have been studied [5, 10, 21, 22]. Liu et al. [9] proposed an SEIRS epidemic model based on scale-free networks. They assumed that the active contact number of each vertex in the model was constant or proportional to its degree, and obtained its epidemic threshold respectively by analytical method. Zhu et al. proposed a generalized epidemic model of a complex heterogeneous network in [24]. In order to explain the simulation results on the network theoretically, the epidemic dynamics was analyzed mathematically using the method of meanfield approximation. Further, they studied the stability of the disease-free equilibrium and the endemic equilibrium. Liu et al. [8] discussed the SEIR model of new epidemic with discrete delay in complex networks. And the basic reproduction number $R_{0}$ of the model was given. Zhang et al. [19] established a stochastic SEIR epidemic dynamic model with migration and human awareness in complex networks.

\footnotetext{
*Corresponding author

Email addresses: 864415225@qq. com (Hairong Yan), ljxsmile1@163.com (Jinxian Li)
}

doi: $10.22436 /$ jnsa.014.04.02

Received: 2020-10-17 Revised: 2020-11-27 Accepted: 2020-12-02 
In fact, many diseases have a latent period. For example, the latent period of epidemic cholera is about 1-3 days, diphtheria 2-4 days, scarlatina 2-5 days, COVID-19 5-6 days, chincough 7-10 days, poliomyelitis 7-14 days, measles 10-11 days, hepatitis B virus 100 days, HIV/AIDS 9-10 years and so on [9]. Therefore, in the early days of the COVID-19 outbreak, many researchers studied it using the SEIR model [14, 23]. Radulescu et al. [15] constructed and analyzed the adaptability of specific dynamic compartments and epidemic parameters for COVID-19 transmission in age-heterogeneous communities to traditional SEIR epidemic models. He et al. [4] built a SEIR epidemic model of COVID-19 based on general control strategies, such as hospitals, quarantine and external input.

Since infectious with COVID-19 do not develop symptoms during the latent period, we cannot quarantine them. Therefore, the infectivity and quarantine may depend on the time elapsed since infection (also called infection age). At present, many models with infection age have been established [6, 7, 20]. Cator et al. [2] considered the SIS model on an arbitrary network, which took into account non-exponential distributions during infection and recovery and established criteria for calculating epidemic thresholds. Röst et al. [16] proposed the generalized mean-field and pairwise model for non-Markovian SIR epidemic model. Meanwhile, it obtained the pairwise reproduction number and the general expression for the final size of this model. In addition, it also noted that distributions with the same mean but smaller variance led to epidemics that grew faster initially.

In order to better describe the dynamics of disease, we will model the spread of disease in heterogeneous networks. Chen et al. [3] took into account that not only could nodes be in states S and I, but also they belong to different connectivity classes $k$ and built a mean-field equations which was degreedependent. They showed that basic reproduction number and the disease dynamics relied not only on the network structure, but also on an age-dependent factor. Miller [12,13] and Volz [18] provided effective ways which were based on the generating functions to describe the spread of disease in heterogeneous networks. Sherborne et al. [17] extended the edge-based compartment model $[12,13]$ to the case where the transmission and recovery process of infectious diseases were driven by a general independent probability distribution. They calculated the final epidemic size of this system, and strictly proved that the edge-based compartmental model and the message passing model were equivalent in the general independent transmission and recovery process.

In this paper, based on the Volz network SIR model [18], quarantine node were introduced to construct the network SIQR model, in which both the infection process and the quarantine process are related to infection age. This article focuses more on the effects of the infection time or the quarantine time distribution on the disease dynamics. The remainder of this paper is organized as follows. In Section 2, we established an SIQR-Epidemic transmission model for the non-Markovian infection process and quarantine process in a heterogeneous complex network. We derive the epidemic threshold of the model in Section 3. In Section 4, a wealth of numerical simulations has been given to study the effects of different infection or quarantine time distributions (Gamma distribution, Uniform distribution, and Weibull distribution) on the disease dynamics. Finally, conclusions and discussions are given in Section 5.

\section{The SIQR epidemic model with infection age}

In this section, we will establish an SIQR-epidemic spreading model with infection age in a complex network. Firstly, we introduce the epidemic propagation mechanism.

\subsection{Epidemic propagation mechanism}

In this paper, the nodes are divided into four groups: susceptible $(\mathcal{S})$, infectious $(\mathcal{J})$, quarantine $(Q)$, or recovered $(\mathcal{R})$. We use $S, I, Q$, and $R$ to denote the fraction of nodes in the sets $\mathcal{S}, \mathcal{J}, \mathcal{Q}$, and $\mathcal{R}$, respectively. And $i(t, a)$ is introduced as the fraction of the density of infected nodes with respect to 
infection age $a$ at time $t$, so we have $I(t)=\int_{0}^{\infty} i(t, a) d a$. In addition, they satisfy the normalization condition: $\mathrm{S}(\mathrm{t})+\mathrm{I}(\mathrm{t})+\mathrm{Q}(\mathrm{t})+\mathrm{R}(\mathrm{t})=1$.

The SIQR epidemic spreading process is shown in Figure 1.

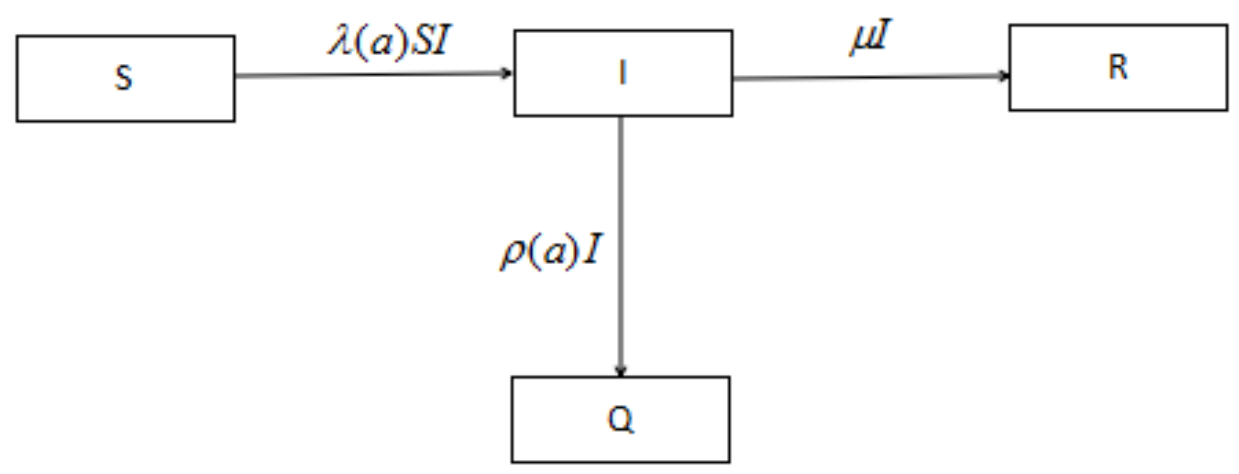

Figure 1: SIQR spreading process.

Moreover, the SIQR epidemic spreading rules are summarized as follows. (1) When a susceptible node comes into contact with an infectious node, the probability that a susceptible node becomes an infectious node is $\lambda(a)$, that is, the infectious rate. (2) An infectious node may become a recovered node directly with probability $\mu$, or it can become a quarantine node with probability $\rho(a)$ and then a recovered node with probability $\omega$.

Since the infection process and the quarantine process are considered non-Markovian, we assume that cumulative distribution function are $F_{1}(a)$ and $F_{2}(a)$, probability density functions are $f_{1}(a)$ and $f_{2}(a)$, associated survival functions are $\xi_{1}(a)=1-F_{1}(a)$ and $\xi_{2}(a)=1-F_{2}(a)$. According to the definition of literature $[6,16,20]$, hazard functions are obtained as

$$
\lambda(a)=-\frac{\dot{\xi_{1}}(a)}{\xi_{1}(a)}=\frac{f_{1}(a)}{\xi_{1}(a)}, \quad \rho(a)=-\frac{\dot{\xi_{2}}(a)}{\xi_{2}(a)}=\frac{f_{2}(a)}{\xi_{2}(a)} .
$$

And $\lambda(a)$ and $\rho(a)$ are bounded on the interval $R_{+}=[0, \infty)$.

\subsection{Our model}

In this section, we follow the notations and symbols in the literature [18]. Here is a summary. To model spreading progress in a heterogeneous network, we consider a closed and mixed population $G=(V, E)$ with $N$ individuals, where $V=\left\{v_{1}, v_{2}, \ldots, v_{N}\right\}$ is the set of vertices representing the individuals and $E$ is the set of edges representing the connections between the individuals. We also assume that contacts are symmetric, that is, if an edge $\left(v_{1}, v_{2}\right) \in \mathrm{E}$ connects $v_{1}$ to $v_{2}$, then an edge also connects $v_{2}$ to $v_{1}$. Although the network is undirected (i.e., any two neighboring vertices can infect each other), we wish to keep track of who infects who. Therefore, for each edge $\left(v_{1}, v_{2}\right) \in E$, we define two arcs as the ordered pairs $\left(v_{1}, v_{2}\right)$ and $\left(v_{2}, v_{1}\right)$. The first and second elements in the ordered pair $\left(v_{1}, v_{2}\right)$ are frequently called the ego and the alter, respectively [18]. Let $p_{k}$ be the degree distribution ( $p_{k}$ is the probability that a random node has $k$ degrees). Then the probability generating functions (PGFs) of the degree distribution is given by

$$
g(x)=p_{0}+p_{1} x+p_{2} x^{2}+p_{3} x^{3}+\cdots .
$$

Let $\langle k\rangle$ be the average degree, and we get $\langle k\rangle=\sum_{k}\left(k p_{k}\right)=g^{\prime}(1)$.

For convenience, some notation are given in Table 1. 
Table 1: Symbol.

\begin{tabular}{|c|c|}
\hline Symbol & Description \\
\hline $\mathcal{A}_{X}$ & Set of arcs (ego, alter) with ego in set X \\
\hline$M_{X}$ & Fraction of arcs in set $\mathcal{A}_{X}$ \\
\hline $\mathcal{A}_{\mathrm{XY}}$ & Set of arcs (ego, alter $)$ s.t. ego $\in X$ and alter $\in Y$ \\
\hline$M_{X Y}$ & Fraction of arcs in set $\mathcal{A}_{\mathrm{XY}}$ \\
\hline$\theta$ & The fraction of degree one nodes that remain susceptible at time $t$ \\
\hline$p_{i}(t, a)$ & $\begin{array}{l}\text { The probability that an arc with a susceptible } \\
\text { ego has an infectious alter, where the infectious alter has infection age } a \text { at time } t\end{array}$ \\
\hline$p_{S}(t)$ & The probability that an arc with a susceptible ego has a susceptible alter at time $t$ \\
\hline
\end{tabular}

By defining these symbols, we can get

$$
\begin{aligned}
p_{i}(t, a) & =M_{S i}(t, a) / M_{S}(t), \\
p_{S}(t) & =M_{S S}(t) / M_{S}(t), \\
p_{I}(t) & =\int_{0}^{\infty} p_{i}(t, a) d a .
\end{aligned}
$$

The SIQR model is summarized as follow. The detailed derivation process is given in Appendix A.

$$
\left\{\begin{array}{l}
\dot{\theta}=-\theta \int_{0}^{\infty} \lambda(a) p_{i}(t, a) d a \\
\dot{p}_{S}(t)=p_{S}(t)\left(1-\theta \frac{g^{\prime \prime}(\theta)}{g^{\prime}(\theta)}\right) \int_{0}^{\infty} \lambda(a) p_{i}(t, a) d a \\
\left(\frac{\partial}{\partial t}+\frac{\partial}{\partial a}\right) p_{i}(t, a)=-(\lambda(a)+\rho(a)+\mu) p_{i}(t, a)+p_{i}(t, a) \int_{0}^{\infty} \lambda(a) p_{i}(t, a) d a
\end{array}\right.
$$

To facilitate numerical simulation, we supplement the model with

$$
\left\{\begin{array}{l}
S=g(\theta), \\
\left(\frac{\partial}{\partial t}+\frac{\partial}{\partial a}\right) i(t, a)=-\mu i(t, a)-\rho(a) i(t, a) .
\end{array}\right.
$$

The boundary condition of model (2.6) is

$$
p_{i}(t, 0)=\frac{p_{S}(t) \theta g^{\prime \prime}(\theta)}{g^{\prime}(\theta)} \int_{0}^{\infty} \lambda(a) p_{i}(t, a) d a .
$$

\subsection{The initial conditions}

Because only a small number of nodes in the network are selected uniformly at randomly and infected at the initial moment, the probability of connecting two initially infected nodes is low. If the probability that a node is selected as an initially infected is $\epsilon$, that is, $\mathrm{I}(0)=\epsilon$. The initial infection density of various infection age obey the Uniform distribution, which is assumed to have the probability density function $\varphi(a)$. So we have $\int_{0}^{A} \varphi(a) d a=1$, where $A<\infty$ is the maximal infection age. We assume that the initial infected nodes are not interconnected. Therefore $\int_{0}^{\infty} M_{S i}(0, a) d a \approx \int_{0}^{\infty} M_{i}(0, a) d a=I(0)=\epsilon$. There are no recovered nodes initially, so $M_{S}(0)=1-\int_{0}^{\infty} M_{S i}(0, a) d a=1-\epsilon$. Finally, $M_{S S}(0)=$ $M_{S}(0)-\int_{0}^{\infty} M_{S i}(0, a) d a=1-2 \epsilon$. Hence, we have the following initial conditions:

$$
\theta(0)=1-\epsilon, \quad p_{i}(0, a)=\frac{M_{S i}(0, a)}{M_{S}(0)}=\frac{\epsilon \varphi(a)}{1-\epsilon}, \quad p_{S}(0)=\frac{M_{S S}(0)}{M_{S}(0)}=\frac{1-2 \epsilon}{1-\epsilon} .
$$

Using the method of characteristics, $p_{i}(t, \infty)=p_{i}(0, \infty)=0$, model (2.6) is converted into the following integro-differential equations

$$
\begin{aligned}
\dot{\theta} & =-\theta \int_{0}^{\infty} \lambda(a) p_{i}(t, a) d a \\
\dot{p}_{S}(t) & =p_{S}(t)\left(1-\theta \frac{g^{\prime \prime}(\theta)}{g^{\prime}(\theta)}\right) \int_{0}^{\infty} \lambda(a) p_{i}(t, a) d a,
\end{aligned}
$$




$$
\begin{aligned}
\dot{p}_{I}(t)= & \frac{p_{S}(t) \theta g^{\prime \prime}(\theta)}{g^{\prime}(\theta)} \int_{0}^{\infty} \lambda(a) p_{i}(t, a) d a-\mu p_{I}(t) \\
& -\left(1-p_{I}(t)\right) \int_{0}^{\infty} \lambda(a) p_{i}(t-a, a) d a \\
& \times \int_{0}^{t} \frac{p_{S}(t-a) \theta g^{\prime \prime}(\theta)}{g^{\prime}(\theta)} e^{-\int_{0}^{a} \mu d s+\int_{0}^{a} \int_{0}^{\infty} \lambda(u) p_{i}(t-a+s, u) d u d s} f_{1}(a) \xi_{2}(a) d a \\
& -\left(1-p_{I}(t)\right) \int_{t}^{\infty} \frac{\epsilon \varphi(a-t)}{1-\epsilon} e^{-\int_{a-t}^{a} \mu d s+\int_{0}^{t} \int_{0}^{\infty} \lambda(u) p_{i}(s, u) d u d s} \frac{f_{1}(a)}{\xi_{1}(a-t)} \frac{\xi_{2}(a)}{\xi_{2}(a-t)} d a \\
& -\int_{0}^{\infty} \lambda(a) p_{i}(t-a, a) d a \\
& \times \int_{0}^{t} \frac{p_{S}(t-a) \theta g^{\prime \prime}(\theta)}{g^{\prime}(\theta)} e^{-\int_{0}^{a} \mu d s+\int_{0}^{a} \int_{0}^{\infty} \lambda(u) p_{i}(t-a+s, u) d u d s} \xi_{1}(a) f_{2}(a) d a \\
& -\int_{t}^{\infty} \frac{\epsilon \varphi(a-t)}{1-\epsilon} e^{-\int_{a-t}^{a} \mu d s+\int_{0}^{t} \int_{0}^{\infty} \lambda(u) p_{i}(s, u) d u d s} \frac{\xi_{1}(a)}{\xi_{1}(a-t)} \frac{f_{2}(a)}{\xi_{2}(a-t)} d a .
\end{aligned}
$$

The calculation process is given in Appendix B.

\section{Epidemic threshold}

Next we will analyze the threshold which means the critical value. Below a threshold, the disease disappears. The number of susceptible becoming infected in a small time interval is proportional to $\dot{p}_{I}$. Therefore, if $\dot{p}_{I}(t=0)<0$, a disease would inevitably disappear without reaching a fraction of the population. The epidemic threshold occurs where

$$
\begin{aligned}
\dot{p}_{I}(t=0)=0= & \frac{p_{S}(0) \theta g^{\prime \prime}(\theta)}{g^{\prime}(\theta)} \int_{0}^{\infty} \lambda(a) p_{i}(0, a) d a-\mu p_{I}(0) \\
& -\left(1-p_{I}(0)\right) \int_{0}^{\infty} \frac{\epsilon \varphi(a)}{1-\epsilon} \frac{f_{1}(a)}{\xi_{1}(a)} d a-\int_{0}^{\infty} \frac{\epsilon \varphi(a)}{1-\epsilon} \frac{f_{2}(a)}{\xi_{2}(a)} d a .
\end{aligned}
$$

Applying the initial conditions above and Eq. (2.1) into $\dot{\mathrm{p}}_{\mathrm{I}}$ and considering $\epsilon \ll 1$ gives

$$
\begin{aligned}
\dot{p}_{I}(t=0)= & \frac{1-2 \epsilon}{1-\epsilon}(1-\epsilon) \frac{g^{\prime \prime}(\theta)}{g^{\prime}(\theta)} \int_{0}^{\infty} \lambda(a) \frac{\epsilon \varphi(a)}{1-\epsilon} d a-\mu \int_{0}^{\infty} \frac{\epsilon \varphi(a)}{1-\epsilon} d a \\
& -\left(1-\int_{0}^{\infty} \frac{\epsilon \varphi(a)}{1-\epsilon} d a\right) \int_{0}^{\infty} \frac{\epsilon \varphi(a)}{1-\epsilon} \lambda(a) d a-\int_{0}^{\infty} \frac{\epsilon \varphi(a)}{1-\epsilon} \rho(a) d a \\
\approx & \epsilon\left(\frac{g^{\prime \prime}(1)}{g^{\prime}(1)} \int_{0}^{\infty} \varphi(a) \lambda(a) d a-\mu-\int_{0}^{\infty} \varphi(a) \lambda(a) d a-\int_{0}^{\infty} \varphi(a) \rho(a) d a\right) .
\end{aligned}
$$

Then $\dot{p}_{I}(t=0)>0$ leads to

$$
\frac{g^{\prime \prime}(1)}{g^{\prime}(1)} \int_{0}^{\infty} \varphi(a) \lambda(a) d a>\mu+\int_{0}^{\infty} \varphi(a) \lambda(a) d a+\int_{0}^{\infty} \varphi(a) \rho(a) d a,
$$

i.e.,

Therefor the threshold is

$$
\frac{\int_{0}^{\infty} \varphi(a) \lambda(a) d a}{\mu+\int_{0}^{\infty} \varphi(a) \rho(a) d a}>\frac{g^{\prime}(1)}{g^{\prime \prime}(1)-g^{\prime}(1)} .
$$

$$
\left(\frac{\int_{0}^{\infty} \varphi(a) \lambda(a) d a}{\mu+\int_{0}^{\infty} \varphi(a) \rho(a) d a}\right)^{*}=\frac{g^{\prime}(1)}{g^{\prime \prime}(1)-g^{\prime}(1)}
$$

\section{Simulations}

In this section, we will carry out numerical simulation to analyze the effects of infection age on disease spreading.

We carry out numerical simulations of model (2.6) in the truncated Poisson network and the truncatedPower-law network. So $p_{k}$ is represented as follows 


$$
p_{k}= \begin{cases}1-\sum_{k=2}^{30} \frac{\alpha^{k} e^{-\alpha}}{k !}, & k=1, \\ \frac{\alpha^{k} e^{-\alpha}}{k !}, & k=2,3,4, \ldots, 30,\end{cases}
$$

or

$$
p_{k}= \begin{cases}1-\sum_{k=2}^{30}(43 / 90) k^{(-1.2)}, & k=1, \\ (43 / 90) k^{(-1.2)}, & k=2,3,4, \ldots, 30 .\end{cases}
$$

Furthermore, both averaged degrees of the networks above are 8.3382, i.e., $\langle k\rangle=8.3382$. And we're given $\mathrm{N}=10^{4}$. According to the CM model proposed by $\operatorname{Volz}(2008)$ [18], we get the new network distribution.

To describe the scale of the disease, we adopt the cumulative epidemic incidence, defined as the fraction of infectious or quarantine or recovered nodes (Volz 2008 [18]), which is denoted as J. Then we have

$$
J=1-g(\theta) .
$$

\subsection{Markovian transmission and non-Markovian quarantine}

Here, we use three common distributions, Gamma, Uniform or Weibull distributions, to describe the quarantine process $[6,20]$. Let $X$ be the quarantine time, which is a random variable. So $P(X \leqslant t)$ is the probability that the quarantine time is less than or equal to $t$.

(1) If $X$ obeys the Gamma distribution with shape $a_{1}$ and scale $b_{1}$, denoted as $G\left(a_{1}, b_{1}\right)$. The probability density function is

$$
f_{2}(x)= \begin{cases}\frac{x^{a_{1}-1} e^{-\frac{x}{b_{1}}}}{\Gamma\left(a_{1}\right) b_{1}^{a_{1}}}, & x \geqslant 0 \\ 0, & x<0\end{cases}
$$

the quarantine rate is

$$
\rho(a)=\frac{a^{a_{1}-1} e^{-\frac{a}{b_{1}}}}{b_{1}^{a_{1}}\left(\Gamma\left(a_{1}\right)-\int_{0}^{\frac{a}{b_{1}}} x^{a_{1}-1} e^{-x} d t\right)} \text { for } a \geqslant 0,
$$

where $\Gamma\left(a_{1}\right)$ is Gamma function. The expected value is $E(X)=a_{1} b_{1}$, and the variance is $\sigma^{2}(X)=$ $a_{1} b_{1}^{2}$. If $E(X)=1$, then the Gamma distribution is defined by one parameter $a_{1}$. Hence we denote it as $G\left(a_{1}\right)$.

(2) If $X$ obeys the Uniform distribution on the interval $\left[a_{2}, b_{2}\right]$, denoted as $U\left(a_{2}, b_{2}\right)$. The probability density function is

$$
f_{2}(x)= \begin{cases}\frac{1}{b_{2}-a_{2}}, & a_{2} \leqslant x \leqslant b_{2} \\ 0, & \text { otherwise }\end{cases}
$$

the quarantine rate is

$$
\rho(a)=\frac{1}{b_{2}-a} \text { for } a \in\left[a_{2}, b_{2}\right],
$$

where $b_{2}>a_{2} \geqslant 0$. The expected value is $E(X)=\frac{a_{2}+b_{2}}{2}$, and the variance is $\sigma^{2}(X)=\frac{\left(b_{2}-a_{2}\right)^{2}}{12}$. If $E(X)=1$, then the Uniform distribution is defined by one parameter $a_{2}$. Hence we denote it as $\mathrm{U}\left(\mathrm{a}_{2}\right)$.

(3) If $X$ obeys the Weibll distribution with shape $a_{3}$ and scale $b_{3}$, denoted as $W\left(a_{3}, b_{3}\right)$. The probability density function is

$$
f_{2}(x)= \begin{cases}\frac{a_{3}}{b_{3}}\left(\frac{x}{b_{3}}\right)^{a_{3}-1} e^{-\left(\frac{x}{b_{3}}\right)^{a_{3}},}, & x \geqslant 0, \\ 0, & x<0,\end{cases}
$$


the quarantine rate is

$$
\rho(a)=\frac{a_{3}}{b_{3}}\left(\frac{a}{b_{3}}\right)^{a_{3}-1} \text { for } a \geqslant 0 .
$$

The expected value is $E(X)=b_{3} \Gamma\left(1+\frac{1}{a_{3}}\right)$, and the variance is $\sigma^{2}(X)=b_{3}^{2}\left[\Gamma\left(1+\frac{2}{a_{3}}\right)-\left(\Gamma\left(1+\frac{1}{a_{3}}\right)\right)^{2}\right]$. If $E(X)=1$, then the Weibull distribution is defined by one parameter $a_{3}$. Hence we denote it as $W\left(a_{3}\right)$.

In this subsection, if not otherwise specified, the numerical simulations of model (2.6) are performed with $\lambda=0.3, \mu=0.1, \mathrm{~N}=10^{4}$, the initial conditions given in (2.7) with $\epsilon=0.001, p_{k}$ given in (4.1) or (4.2) and $\rho(\mathrm{a})$ given above in different cases. detail.

Next, we will study the effect of the quarantine time distribution on the spread of disease in more

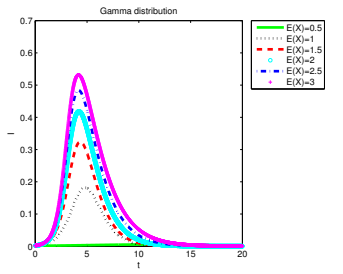

(a)

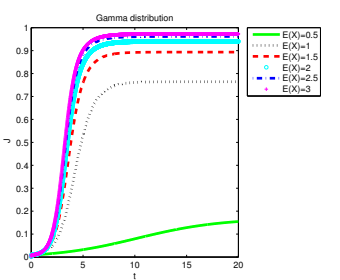

(d)

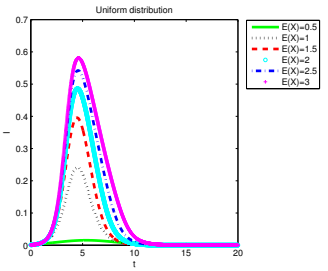

(b)

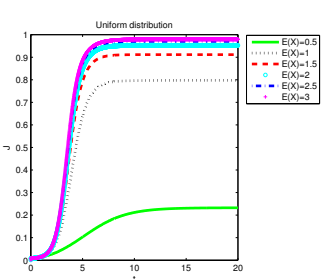

(e)

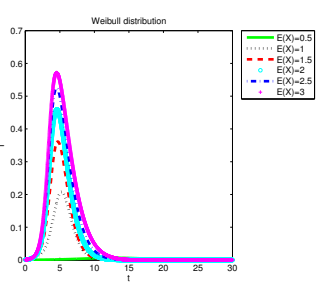

(c)

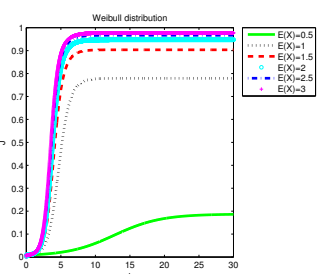

(f)

Figure 2: In the network with truncated Poisson degree distribution, the time series of the number of the infection density with the different quarantine time distributions: (a) Gamma distribution $G\left(a_{1}, b_{1}\right)$, where $a_{1}=1.5$, (b) Uniform distribution $\mathrm{U}\left(\mathrm{a}_{2}, \mathrm{~b}_{2}\right)$, where $\mathrm{a}_{2}=0.1$, and (c) Weibull distribution $\mathrm{W}\left(\mathrm{a}_{3}, \mathrm{~b}_{3}\right)$, where $a_{3}=1.5$, respectively. In the network with Poisson degree distribution, the time series of the cumulative epidemic incidence with the different quarantine time distributions: (d) Gamma distribution $G\left(a_{1}, b_{1}\right)$, where $a_{1}=1.5$, (e) Uniform distribution $U\left(a_{2}, b_{2}\right)$, where $a_{2}=0.1$, and (f) Weibull distribution $W\left(a_{3}, b_{3}\right)$, where $a_{3}=1.5$, respectively.

Figures 2 and 3 show the temporal evolutions of the infection density and the cumulative epidemic incidence for the different quarantine time distributions and in the network with the truncated Poisson degree distribution and the truncated Power-law degree distribution, respectively. Let $a_{1}=1.5, a_{2}=$ $0.1, a_{3}=1.5$ and different expectations of quarantine time $E(X)$ are given. The results show that the increases in the expectations of quarantine time bring the larger peaks and the larger cumulative epidemic incidence. Therefore, timely quarantine can reduce the peak of infection density and the cumulative epidemic incidence.

In Figure 4, the temporal evolutions of the infection density and the cumulative epidemic incidence for the different quarantine time distributions with different parameters $a_{i}(i=1,2,3)$ are given. Since $E(X)=1$, the variance can be looked as the function of $a_{i}$. And it is easy to see that this function is decreasing. So greater $a_{i}$ leads to smaller variance. The results in Figure 4 show that, with given $a_{i}$, the infection density goes up to the peak first and then goes down to zero, the cumulative epidemic incidence is rising to the peak first and then remains constant. Furthermore, greater variances, i.e., smaller $a_{i}$, will lead to the smaller peaks and cumulative epidemic incidence. 


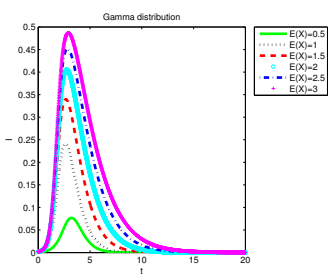

(a)

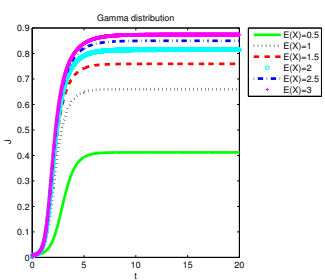

(d)

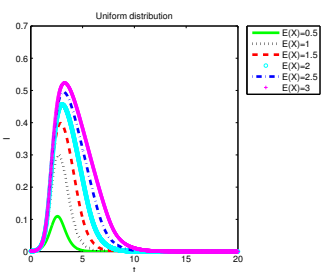

(b)

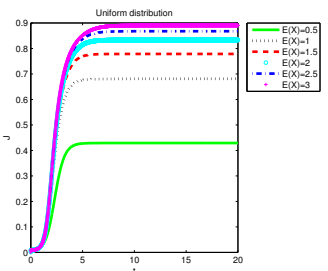

(e)

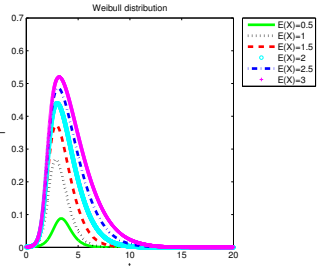

(c)

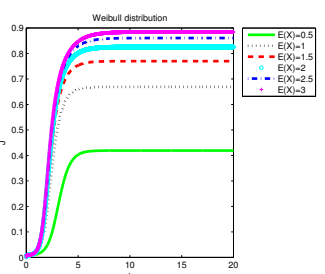

(f)

Figure 3: In the network with truncated Power-law degree distribution, the time series of the number of the infection density with the different quarantine time distributions: (a) Gamma distribution $G\left(a_{1}, b_{1}\right)$, where $a_{1}=1.5$, (b) Uniform distribution $\mathrm{U}\left(\mathrm{a}_{2}, \mathrm{~b}_{2}\right)$, where $\mathrm{a}_{2}=0.1$, and (c) Weibull distribution $\mathrm{W}\left(\mathrm{a}_{3}, \mathrm{~b}_{3}\right)$, where $a_{3}=1.5$, respectively; In the network with Power-law degree distribution, the time series of the cumulative epidemic incidence with the different quarantine time distributions: (d) Gamma distribution $G\left(a_{1}, b_{1}\right)$, where $a_{1}=1.5$, (e) Uniform distribution $U\left(a_{2}, b_{2}\right)$, where $a_{2}=0.1$, and (f) Weibull distribution $W\left(a_{3}, b_{3}\right)$, where $a_{3}=1.5$, respectively.

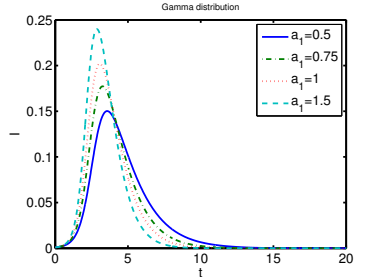

(a)

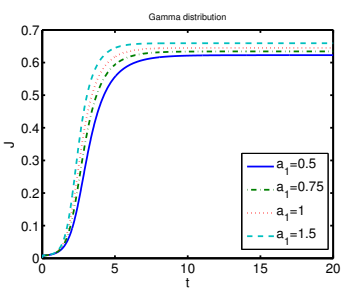

(d)

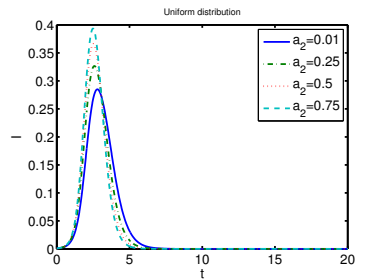

(b)

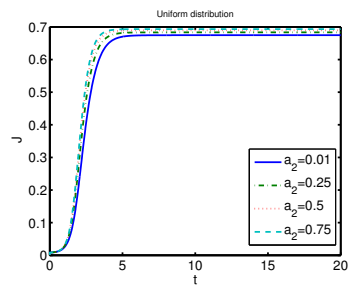

(e)

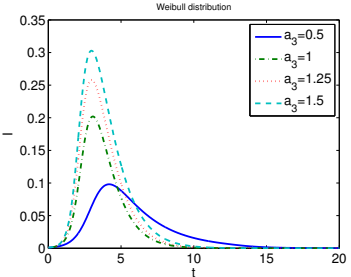

(c)

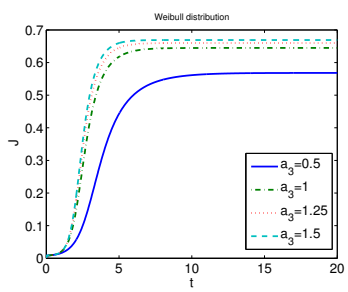

(f)

Figure 4: The time series of the infection density for the different quarantine time distributions with different parameters $a_{i}$ $(i=1,2,3)$ : (a) Gamma distribution; (b) Uniform distribution; (c) Weibull distribution. The time series of the cumulative epidemic incidence for the different quarantine time distributions with different parameters $a_{i}(i=1,2,3)$ : (d) Gamma distribution; (e) Uniform distribution; (f) Weibull distribution.

\subsection{Non-Markovian transmission and Markovian quarantine}

In this subsection, we assume that the infection time distribution $f_{1}(a)$ is one of three different distributions, namely Gamma distribution $\mathrm{G}\left(\mathrm{a}_{4}, \mathrm{~b}_{4}\right)$, Uniform distribution $\mathrm{U}\left(\mathrm{a}_{5}, \mathrm{~b}_{5}\right)$ and Weibull distribution $W\left(a_{6}, b_{6}\right)$. Let $Y$ be the infection time, which is a random variable. We can get the infection rates of the 
three distributions

$$
\begin{aligned}
& \lambda(a)=\frac{a^{a_{4}-1} e^{-\frac{a}{b_{4}}}}{b_{4}^{a_{4}}\left(\Gamma\left(a_{4}\right)-\int_{0}^{\frac{a}{b_{4}}} \chi^{a_{4}-1} e^{-x} d t\right)} \text { for } a \geqslant 0, \\
& \lambda(a)=\frac{1}{b_{5}-a} \text { for } a \in\left[a_{5}, b_{5}\right], \\
& \lambda(a)=\frac{a_{6}}{b_{6}}\left(\frac{a}{b_{6}}\right)^{a_{6}-1} \text { for } a \geqslant 0,
\end{aligned}
$$

respectively.

If not otherwise specified, the numerical simulations of model (2.6) are performed with $\rho=0.2$, $\mu=0.1, \mathrm{~N}=10^{4}$, the initial conditions given in (2.7) with $\epsilon=0.001, \mathrm{p}_{\mathrm{k}}$ given in (4.1) or (4.2) and $\lambda(\mathrm{a})$ given above in different cases.

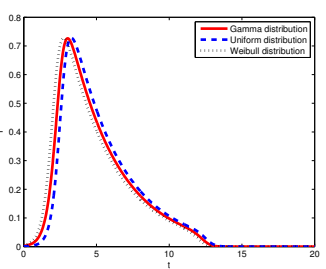

(a)

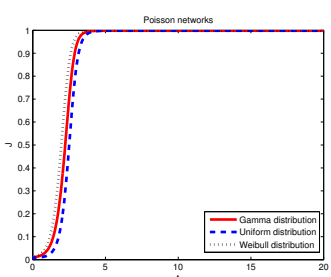

(d)

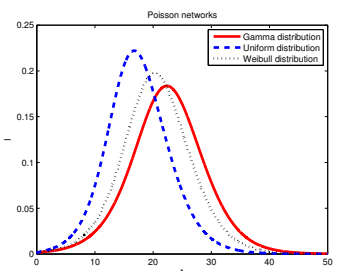

(g)

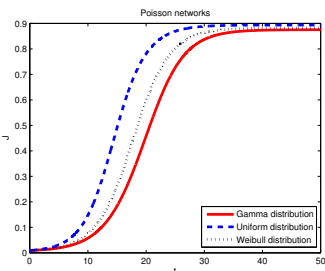

(j)

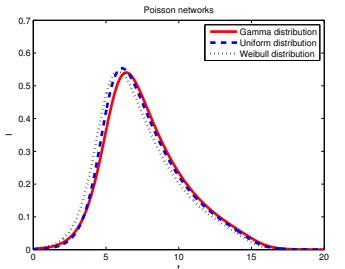

(b)

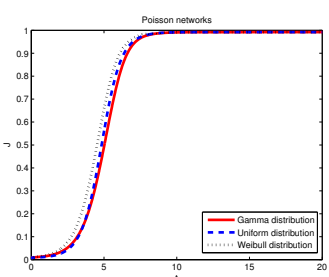

(e)

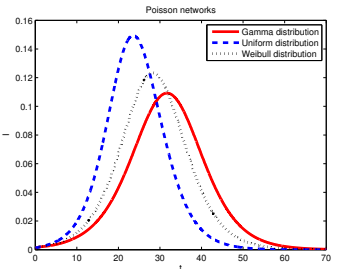

(h)

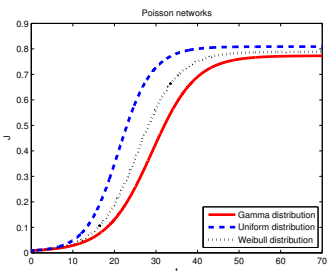

$(\mathrm{k})$

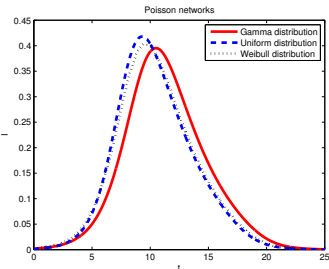

(c)

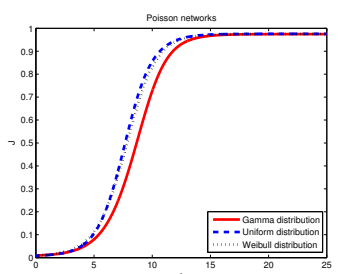

(f)

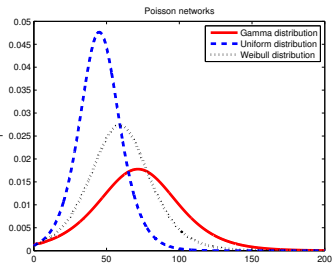

(i)

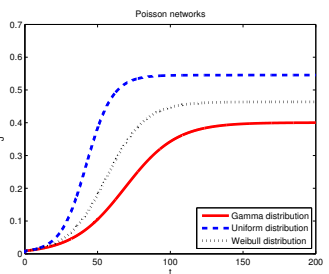

(1)

Figure 5: Numerical simulations of (2.6) in the network with truncated Poisson degree distribution and for the different infection time distributions. The parameters of the distributions are set to have the same mean and variance. The expectation and the variances are: (a), (d) $E(Y)=1, \sigma^{2}(Y)=0.2732$, (b), (e) $E(Y)=2, \sigma^{2}(Y)=1.0930,(c),(f) E(Y)=3, \sigma^{2}(Y)=2.4592,(g),(j) E(Y)=5$, $\sigma^{2}(Y)=6.8314,(h),(k) E(Y)=6, \sigma^{2}(Y)=9.8372$, and (i), (l) $E(Y)=8, \sigma^{2}(Y)=17.4882$. 


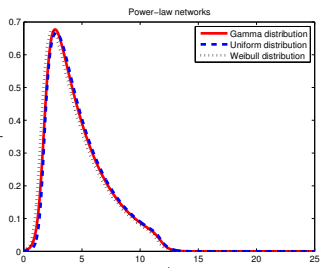

(a)

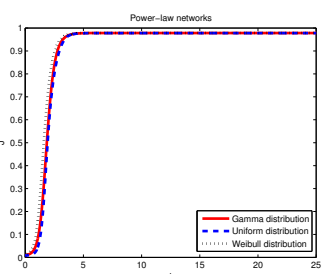

(d)

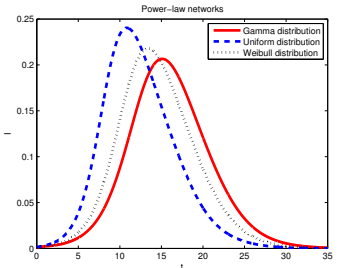

(g)

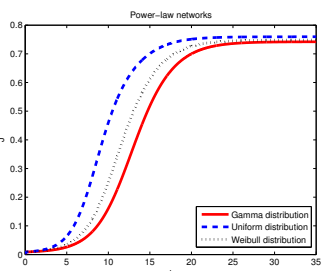

(j)

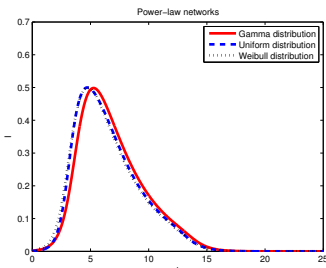

(b)

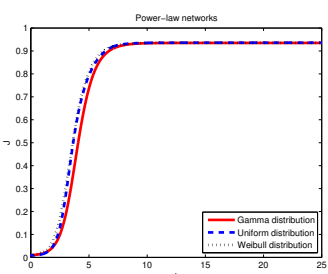

(e)

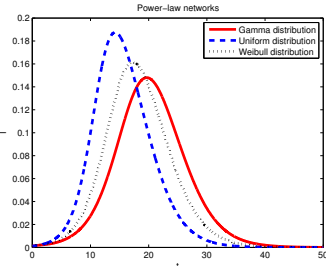

(h)

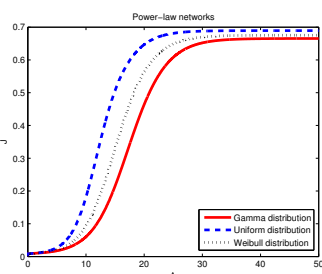

(k)

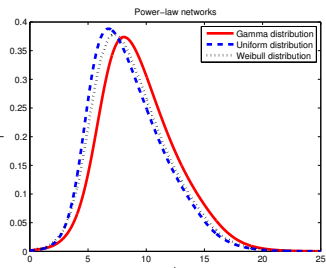

(c)

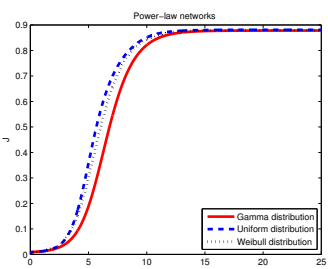

(f)

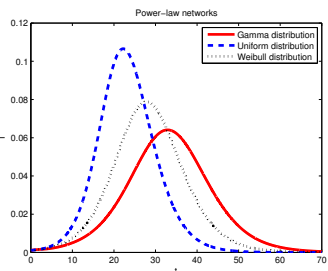

(i)

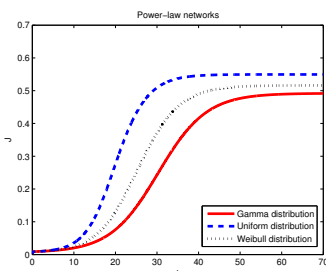

(1)

Figure 6: Numerical simulations of (2.6) in the network with truncated Power-law degree distribution and for the different infection time distributions. The parameters of the distributions are set to have the same mean and variance. The expectation and the variances are: (a), (d) $E(Y)=1, \sigma^{2}(Y)=0.2732,(b),(e) E(Y)=2, \sigma^{2}(Y)=1.0930,(c),(f) E(Y)=3, \sigma^{2}(Y)=2.4592,(g),(j)$ $\mathrm{E}(\mathrm{Y})=5, \sigma^{2}(\mathrm{Y})=6.8314,(\mathrm{~h}),(\mathrm{k}) \mathrm{E}(\mathrm{Y})=6, \sigma^{2}(\mathrm{Y})=9.8372$, and $(\mathrm{i}),(\mathrm{l}) \mathrm{E}(\mathrm{Y})=8, \sigma^{2}(\mathrm{Y})=17.4882$.

Figures 5 and 6 show the temporal evolutions of the infection density and the cumulative epidemic incidence for the different infection time distributions in the network with the truncated Poisson degree distribution and the truncated Power-law degree distribution, respectively. The parameters of the distributions are set to have the same mean and variance. Comparatively speaking, the infection density and the cumulative epidemic incidence with infection time obey the Uniform distribution has the larger peak, Weibull distribution is the second, Gamma distribution is the smallest.

\section{Conclusion}

In this study, we used the method of generating function to establish the SIQR epidemic model with infection age. Next, we derive the epidemic threshold of the model. Thirdly, we focus on the impact of three different infection or quarantine time distributions on disease transmission. We obtained the following results. 
(1) When $a_{i}(i=1,2,3)$ is fixed and different expectations of quarantine time $E(X)$ are given, the results show that timely quarantine can reduce the peak of infection density and the cumulative epidemic incidence.

(2) Keeping the expectation of quarantine time as one, given $a_{i}(i=1,2,3)$, the infection density goes up to the peak first and then goes down to zero, the cumulative epidemic incidence is rising to the peak first and then remains constant. Furthermore, greater variances, i.e. smaller $a_{i}$, will lead to the smaller peaks and cumulative epidemic incidence.

(3) Comparatively speaking, the infection density and cumulative epidemic incidence with infection time obey the Uniform distribution has the larger peak, Weibull distribution is the second, Gamma distribution is the smallest.

Since many epidemics now quarantine not only the infected but also those who have had close contact with them, this is a question worthy of our further study.

\section{Acknowledgment}

This work is supported by the National Natural Science Foundations of China under Grant (Nos. 11571210, 11971279,11701348,11331009, 11501339, 11101251, 11001157, 11471197).

\section{References}

[1] R. M. Anderson, R. M. May, Infectious diseases of humans: dynamics and control, Oxford University Press, New York, (1992). 1

[2] E. Cator, R. Van de Bovenkamp, P. Van Mieghem, Susceptible-infected-susceptible epidemics on networks with general infection and cure times, Phys. Rev. E, 87 (2013), 7 pages. 1

[3] S. Chen, M. Small, Y. Tao, X. Fu, Transmission Dynamics of an SIS Model with Age Structure on Heterogeneous Networks, Bull. Math. Biol., 80 (2018), 2049-2087. 1

[4] S. He, Y. Peng, K. Sun, SEIR modeling of the COVID-19 and its dynamics, Nonlinear Dyn., 101 (2020), 1667-1680. 1

[5] G. Herzog, R. Redheffer, Nonautonomous SEIRS and Thron models for epidemiology and cell biology, Nonlinear Anal. Real World Appl., 5 (2004), 33-44. 1

[6] W. Jing, Z. Jin, J. Zhang, An SIR pairwise epidemic model with infection age and demography, J. Biol. Dyn., 12 (2018), 486-508. 1, 2.1, 4.1

[7] I. Z. Kiss, G. Röst, Z. Vizi, Generalization of Pairwise Models to non-Markovian Epidemics on Networks, Phys. Rev. Lett., 115 (2015), 5 pages. 1

[8] Q. Liu, H. Li, Global dynamics analysis of an SEIR epidemic model with discrete delay on complex network, Phys. A, 524 (2019), 289-296. 1

[9] J. Liu, T. Zhang, Epidemic spreading of an SEIRS model in scale-free networks, Commun. Nonlinear Sci. Numer. Simul., 16 (2011), 3375-3384. 1

[10] J. Ma, Z. Ma, Epidemic threshold conditions for seasonally forced SEIR models, Math. Biosci. Eng., 3 (2006), 161-172. 1

[11] M. Martcheva, An Introduction to Mathematical Epidemiology, Springer, New York, (2015). 1

[12] J. C. Miller, Epidemic size and probability in populations with heterogeneous infectivity and susceptibility, Phys. Rev. E, 76 (2007), 4 pages. 1

[13] J. C. Miller, A note on a paper by Erik Volz: SIR dynamics in random networks, J. Math. Biol., 62 (2011), 349-358. 1

[14] N. Piovella, Analytical solution of SEIR model describing the free spread of the COVID-19 pandemic, Chaos, Solitons and Fractals, 140 (2020), 6 pages. 1

[15] A. Rădulescu, C. Williams, K. Cavanagh, Management strategies in a seir model of COVID-19 community spread, Physics and Society, (2020), 21 pages. 1

[16] G. Röst, Z. Vizi, I. Z. Kiss, Pairwise approximation for SIR-type network epidemics with non-Markovian recovery, Proc. A., 474 (2018), 21 pages. 1, 2.1

[17] N. Sherborne, J. C. Miller, K. B. Blyuss, I. Z. Kiss, Mean-field models for non-Markovian epidemics on networks, J. Math. Biol., 76 (2018), 755-778. 1

[18] E. Volz, SIR dynamics in random networks with heterogeneous connectivity, J. Math. Biol., 56 (2008), 293-310. 1, 2.2, 4, 5,5

[19] Y. Zhang, Y. Li, Evolutionary Dynamics of Stochastic SEIR Models with Migration and Human Awareness in Complex Networks, Complexity, 2020 (2020), 1-15. 1

[20] J. Zhang, D. Li, W. Jing, Z. Jin, H. Zhu, Transmission dynamics of a two-strain pairwise model with infection age, Appl. Math. Model., 71 (2019), 656-672. 1, 2.1, 4.1 
[21] J. Zhang, Z. Ma, Global dynamics of an SEIR epidemic model with saturating contact rate, Math. Biosci., 185 (2003), 15-32. 1

[22] T. Zhang, Z. Teng, On a nonautonomous SEIRS model in epidemiology, Bull. Math. Biol., 69 (2007), 2537-2559. 1

[23] T. Zhou, Q. Liu, Z. Yang, J. Liao, K. Yang, W. Bai, X. Lu, W. Zhang, Preliminary prediction of the basic reproduction number of the Wuhan novel coronavirus 2019-nCoV, Evid. Based Med., 13 (2020), 3-7. 1

[24] G. Zhu, X. Fu, G. Chen, Spreading dynamics and global stability of a generalized epidemic model on complex heterogeneous networks, Appl. Math. Model., 36 (2012), 5808-5817. 1

\section{Appendix A: The dynamics of SIQR epidemic model in a network}

In this appendix, we will show how to model the disease spreading with infection age, non-Markovian the infection process and non-Markovian the quarantine process with an SIQR-epidemic model involving the variables $\theta, p_{i}$ and $p_{S}$. The method here is an extension of that used in Volz 2008 [18].

Firstly, we will derive the dynamic of $\theta$. The definition of $p_{I}$ shows us that the probability an arc with a susceptible ego has an infectious alter is $p_{I}$. Then a susceptible node with degree $k$ is connected to an infected node with the probability $k p_{I}$. Hence, the hazard for a susceptible node at time $t$ is $k \int_{0}^{\infty} \lambda(a) p_{i}(t, a) d a$. Therefore, let $S_{k}$ represent the probability of nodes with degree $k$ that remain susceptible at time $t$, we have

$$
\dot{S}_{k}=-k S_{k} \int_{0}^{\infty} \lambda(a) p_{i}(t, a) d a .
$$

From Eq. (5.1), we have

$$
S_{k}(t)=\exp \left\{-\int_{0}^{t} k \int_{0}^{\infty} \lambda(a) p_{i}(u, a) d a d u\right\}=\left(\exp \left\{-\int_{0}^{t} \int_{0}^{\infty} \lambda(a) p_{i}(u, a) d a d u\right\}\right)^{k} .
$$

For convenience, we will use the symbol $\theta$ to represent

$$
\theta:=S_{1}=\exp \left\{-\int_{0}^{t} \int_{0}^{\infty} \lambda(a) p_{i}(u, a) d a d u\right\}
$$

From Eq. (5.2), it is clear that $S_{k}(t)=\theta^{k}$. Then the dynamics of $\theta$ is easy to get

$$
\dot{\theta}=-\theta \int_{0}^{\infty} \lambda(a) p_{i}(t, a) d a
$$

Given $\theta$, it is easy to determine the fraction of nodes which remain susceptible at time $t$. Therefore, we have

$$
S=p_{0}+p_{1} S_{1}+p_{2} S_{2}+p_{3} S_{3}+\cdots=p_{0}+p_{1} \theta+p_{2} \theta^{2}+p_{3} \theta^{3}+\cdots=g(\theta)
$$

Furthermore,

$$
\dot{S}=\frac{d}{d t} S=\frac{d}{d t} g(\theta)=\dot{\theta} g^{\prime}(\theta)=-\theta g^{\prime}(\theta) \int_{0}^{\infty} \lambda(a) p_{i}(t, a) d a
$$

Thus, it can be concluded that the density of infected satisfies

$$
\left\{\begin{array}{l}
\left(\frac{\partial}{\partial t}+\frac{\partial}{\partial a}\right) i(t, a)=-\mu i(t, a)-\rho(a) i(t, a), \\
i(t, 0)=\theta g^{\prime}(\theta) \int_{0}^{\infty} \lambda(a) p_{i}(t, a) d a .
\end{array}\right.
$$


Next, we need to derive the dynamic of $p_{i}(t, a)$. According to Eq. (2.3), the dynamic of $p_{i}(t, a)$ follows

$$
\left\{\begin{aligned}
\left(\frac{\partial}{\partial t}+\frac{\partial}{\partial a}\right) p_{i}(t, a) & =\frac{\partial}{\partial t} p_{i}(t, a)+\frac{\partial}{\partial a} p_{i}(t, a) \\
& =\frac{\partial}{\partial t}\left(\frac{M_{S i}(t, a)}{M_{S}(t)}\right)+\frac{\partial}{\partial a}\left(\frac{M_{S i}(t, a)}{M_{S}(t)}\right) \\
& =\frac{1}{M_{S}(t)}\left(\frac{\partial}{\partial t}+\frac{\partial}{\partial a}\right) M_{S i}(t, a)+M_{S i}(t, a)\left(-\frac{M_{S}(t)}{M_{S}^{2}(t)}\right) \\
p_{i}(t, 0) & =\frac{M_{S i}(t, 0)}{M_{S}(t)} .
\end{aligned}\right.
$$

The right side of (5.5) involves $M_{S}, \dot{M}_{S}, M_{S i},\left(\frac{\partial}{\partial t}+\frac{\partial}{\partial a}\right) M_{S i}(t, a), M_{S i}(t, 0)$. According to the definition of $M_{S}(t)$ in Table 1 , we have

$$
M_{S}(t)=\frac{\sum_{k} k p_{k} S_{k}}{\sum_{k} k p_{k}}=\frac{\sum_{k} k p_{k} \theta^{k}}{g^{\prime}(1)}=\frac{\left[\frac{d}{d x} g(\theta x)\right]_{x=1}}{g^{\prime}(1)}=\frac{\theta g^{\prime}(\theta)}{g^{\prime}(1)} .
$$

Then using Eqs. (5.3) and (5.6), we can get the derivative of $M_{S}(t)$,

$$
\dot{M}_{S}(t)=\frac{d}{d t}\left(\frac{\theta g^{\prime}(\theta)}{g^{\prime}(1)}\right)=\frac{\dot{\theta} g^{\prime}(\theta)+\theta \dot{\theta} g^{\prime \prime}(\theta)}{g^{\prime}(1)}=-\frac{\theta}{g^{\prime}(1)}\left(g^{\prime}(\theta)+\theta g^{\prime \prime}(\theta)\right) \int_{0}^{\infty} \lambda(a) p_{i}(t, a) d a .
$$

Furthermore, $M_{S i}(t, a)$ easily follows,

$$
M_{S i}(t, a)=M_{S}(t) \times \frac{M_{S i}(t, a)}{M_{S}(t)}=M_{S}(t) p_{i}(t, a)=\frac{p_{i}(t, a) \theta g^{\prime}(\theta)}{g^{\prime}(1)} .
$$

Similarly, $M_{S S}(t)$ is obtained as follows,

$$
M_{S S}(t)=M_{S}(t) \times \frac{M_{S S}(t)}{M_{S}(t)}=M_{S}(t) p_{S}(t)=\frac{p_{S}(t) \theta g^{\prime}(\theta)}{g^{\prime}(1)}
$$

Table 2: Symbol.

\begin{tabular}{ll}
\hline Symbol & Description \\
\hline$\delta_{X Y}$ & The average excess degree of nodes in state $X$ and selected along an arc $(X, Y)$ \\
$\delta_{X Y}(Z)$ & As $\delta_{X Y}$ but counting only arcs from ego to nodes in state $Z$ \\
\hline
\end{tabular}

To get the dynamic of $M_{S i}$, we need the rearrangement of arcs among sets $A_{S S}$ and $A_{S I}$ as $-\dot{S}$ nodes become infected in a small time interval. Before that, we will introduce the definition of excess degree given in Volz (2008) [18]. Let $\delta_{X Y}$ represents the average degree of nodes in set $X$, selected with probability proportional to the number of arcs to nodes in set $Y$, not counting one arc to nodes of type $Y$. This is commonly called the excess degree of a node. According to the definition of the excess degree, it is easy to get

$$
\delta_{\mathrm{SI}}=\frac{\theta g^{\prime \prime}(\theta)}{g^{\prime}(\theta)}
$$

Then $\delta_{\text {SI }}\left(i_{a}\right) \delta_{S I}(S)$ follow,

$$
\delta_{S I}\left(i_{a}\right)=p_{i}(t, a) \delta_{S I}=\frac{p_{i}(t, a) \theta g^{\prime \prime}(\theta)}{g^{\prime}(\theta)}
$$


where $i_{a}$ is introduced as the fraction of the density of infected nodes with respect to infection age $a$ at time $t$, and

$$
\delta_{S I}(S)=p_{S}(t) \delta_{S I}=\frac{p_{S}(t) \theta g^{\prime \prime}(\theta)}{g^{\prime}(\theta)}
$$

As a fraction $-\dot{S}$ nodes are infected in time $d t, M_{S i}$ is reduced in four way.

Firstly, since there are $\delta_{S I}\left(i_{a}\right)$ infected nodes, not counting the one in the arc, around the newly infected, $M_{S i}$ is reduced at a rate

$$
-\dot{S} \delta_{S I}\left(i_{a}\right) / g^{\prime}(1)=\frac{p_{i}(t, a) \theta^{2} g^{\prime \prime}(\theta)}{g^{\prime}(1)} \int_{0}^{\infty} \lambda(a) p_{i}(t, a) d a
$$

Secondly, because $\delta_{S I}\left(i_{a}\right)$ does not count the arc along which a node was infected, the transmission from the infectious alter to the susceptible ego in this arc brings $M_{S i}$ to decrease at a rate $\lambda(a) M_{S i}(t, a)$. Thirdly, the recovery of the infectious alter in this arc brings $M_{S i}$ to decrease at a rate $\mu M_{S i}(t, a)$. Fourthly, the quarantine of the infectious alter in this arc brings $M_{S i}$ to decrease at a rate $\rho(a) M_{S i}(t, a)$.

At the same time, because there are $\delta_{S I}(S)$ susceptible nodes around the newly infected, the newly increased $M_{S i}$ is

$$
-\dot{S} \delta_{S I}(S) / g^{\prime}(1)=\frac{p_{S}(t) \theta^{2} g^{\prime \prime}(\theta)}{g^{\prime}(1)} \int_{0}^{\infty} \lambda(a) p_{i}(t, a) d a
$$

Using Eqs. (5.10), (5.11), and (5.4), we have

$$
\left\{\begin{array}{l}
\left(\frac{\partial}{\partial t}+\frac{\partial}{\partial a}\right) M_{S i}(t, a)=-(\lambda(a)+\rho(a)+\mu) M_{S i}(t, a)-\frac{p_{i}(t, a) \theta^{2} g^{\prime \prime}(\theta)}{g^{\prime}(1)} \int_{0}^{\infty} \lambda(a) p_{i}(t, a) d a \\
M_{S i}(t, 0)=\frac{p_{S}(t) \theta^{2} g^{\prime \prime}(\theta)}{g^{\prime}(1)} \int_{0}^{\infty} \lambda(a) p_{i}(t, a) d a .
\end{array}\right.
$$

Now substituting Eqs. (5.6), (5.7), (5.8), and (5.12) into Eq. (5.5), we will obtain

$$
\left\{\begin{array}{l}
\left(\frac{\partial}{\partial t}+\frac{\partial}{\partial a}\right) p_{i}(t, a)=-(\lambda(a)+\rho(a)+\mu) p_{i}(t, a)+p_{i}(t, a) \int_{0}^{\infty} \lambda(a) p_{i}(t, a) d a \\
p_{i}(t, 0)=\frac{p_{S}(t) \theta g^{\prime \prime}(\theta)}{g^{\prime}(\theta)} \int_{0}^{\infty} \lambda(a) p_{i}(t, a) d a
\end{array}\right.
$$

According to Eq. (2.4), the dynamic of $p_{S}(t)$ follows,

$$
\dot{p}_{S}(t)=\frac{d}{d t}\left(\frac{M_{S S}(t)}{M_{S}(t)}\right)=\frac{\dot{M}_{S S}(t)}{M_{S}(t)}-\frac{\dot{M}_{S}(t) M_{S S}(t)}{M_{S}^{2}(t)} .
$$

The calculation of $\dot{M}_{S S}(t)$ is very similar to that of $\left(\frac{\partial}{\partial t}+\frac{\partial}{\partial a}\right) M_{S i}(t, a)$. The newly infected nodes have on average $\delta_{S I}(S)$ arcs to other susceptible, so that

$$
\dot{M}_{S S}=\frac{-2 \times(-\dot{S}) \delta_{S I}(S)}{g^{\prime}(1)}=\frac{-2 p_{S}(t) \theta^{2} g^{\prime \prime}(\theta)}{g^{\prime}(1)} \int_{0}^{\infty} \lambda(a) p_{i}(t, a) d a .
$$

Substituting Eqs. (5.6), (5.7), (5.9), and (5.14) into Eq. (5.13), we have

$$
\dot{p}_{S}(t)=p_{S}(t)\left(1-\theta \frac{g^{\prime \prime}(\theta)}{g^{\prime}(\theta)}\right) \int_{0}^{\infty} \lambda(a) p_{i}(t, a) d a .
$$

So we get model (2.6). 


\section{Appendix B: Derivation of equations of second-order motifs for an SIQR model}

In this appendix, we give the derivation of $p_{i}(t, a)$. Let us consider the following first-order PDE:

$$
\left(\frac{\partial}{\partial t}+\frac{\partial}{\partial a}\right) p_{i}(t, a)=-(\lambda(a)+\rho(a)+\mu) p_{i}(t, a)+p_{i}(t, a) \int_{0}^{\infty} \lambda(a) p_{i}(t, a) d a
$$

with boundary condition $p_{i}(t, 0)=\frac{p_{S}(t) \theta g^{\prime \prime}(\theta)}{g^{\prime}(\theta)} \int_{0}^{\infty} \lambda(a) p_{i}(t, a) d a$ and initial condition $p_{i}(0, a)=\frac{\epsilon \varphi(a)}{1-\epsilon}$. In the method of characteristics, it is assumed that the partial differential equation can be expressed as a system of ordinary differential equations along the characteristic curves. The characteristic curves are found by solving the following ordinary differential equations:

$$
\frac{d t}{d \tau}=1, \quad \frac{d a}{d \tau}=1
$$

Let $y(\tau)=p_{i}(t(\tau), a(\tau))$, we have

$$
\frac{d y(\tau)}{d \tau}=-(\lambda(a(\tau))+\rho(a(\tau))+\mu) y(\tau)+y(\tau) \int_{0}^{\infty} \lambda(u) p_{i}(t(\tau), u) d u
$$

with initial conditions

$$
t(0)=t_{0}, \quad a(0)=a_{0}, \quad y(0)=p_{i}\left(t_{0}, a_{0}\right) .
$$

The solution of the system of ordinary differential equations along the characteristic curves is

$$
t(\tau)=t_{0}+\tau, \quad a(\tau)=a_{0}+\tau, \quad y(\tau)=y(0) e^{-\int_{0}^{\tau}[\lambda(a(s))+\rho(a(s))+\mu] d s+\int_{0}^{\tau} \int_{0}^{\infty} \lambda(u) p_{i}(t(s), u) d u d s} .
$$

Furthermore, it has

$$
\begin{aligned}
p_{i}(t, a) & =p_{i}\left(t_{0}, a_{0}\right) e^{-\int_{0}^{\tau}[\lambda(a(s))+\rho(a(s))+\mu] d s+\int_{0}^{\tau} \int_{0}^{\infty} \lambda(u) p_{i}(t(s), u) d u d s} \\
& =p_{i}\left(t_{0}, a_{0}\right) e^{-\int_{0}^{\tau}\left[\lambda\left(a_{0}+s\right)+\rho\left(a_{0}+s\right)+\mu\right] d s+\int_{0}^{\tau} \int_{0}^{\infty} \lambda(u) p_{\mathfrak{i}}\left(t_{0}+s, u\right) d u d s} .
\end{aligned}
$$

If $t>a$, set $a_{0}=0$. Then $\tau=a$ and $t_{0}=t-a$. Substituting these expressions into Eq. (5.16), we obtain the solution when $t>a$

$$
p_{i}(t, a)=p_{i}(t-a, 0) e^{-\int_{0}^{a}[\lambda(s)+\rho(s)+\mu] d s+\int_{0}^{a} \int_{0}^{\infty} \lambda(u) p_{i}(t-a+s, u) d u d s} .
$$

If $t \leqslant a$, set $t_{0}=0$. Then $\tau=t$ and $a_{0}=a-t$. Substituting these expressions into Eq. (5.16), we obtain the solution when $t \leqslant a$

$$
\begin{aligned}
p_{i}(t, a) & =p_{i}(0, a-t) e^{-\int_{0}^{t}[\lambda(a-t+s)+\rho(a-t+s)+\mu] d s+\int_{0}^{t} \int_{0}^{\infty} \lambda(u) p_{i}(s, u) d u d s} \\
& =p_{i}(0, a-t) e^{-\int_{a-t}^{a}[\lambda(s)+\rho(s)+\mu] d s+\int_{0}^{t} \int_{0}^{\infty} \lambda(u) p_{i}(s, u) d u d s} .
\end{aligned}
$$

So finally, the solution of Equation (5.15) can be written in the form

$$
p_{i}(t, a)= \begin{cases}p_{i}(t-a, 0) e^{-\int_{0}^{a}[\lambda(s)+\rho(s)+\mu] d s+\int_{0}^{a} \int_{0}^{\infty} \lambda(u) p_{i}(t-a+s, u) d u d s}, & t>a, \\ p_{i}(0, a-t) e^{-\int_{a-t}^{a}[\lambda(s)+\rho(s)+\mu] d s+\int_{0}^{t} \int_{0}^{\infty} \lambda(u) p_{i}(s, u) d u d s,} & t \leqslant a .\end{cases}
$$

Using the definition and properties of hazard function, we can deduce the following formulae:

$$
e^{-\int_{0}^{a} \lambda(s) d s}=\frac{\xi_{1}(a)}{\xi_{1}(0)}=\xi_{1}(a), \quad e^{-\int_{0}^{a} \rho(s) d s}=\frac{\xi_{2}(a)}{\xi_{2}(0)}=\xi_{2}(a),
$$




$$
\begin{aligned}
e^{-\int_{a-t}^{a} \lambda(s) d s} & =\frac{\xi_{1}(a)}{\xi_{1}(a-t)}, \quad e^{-\int_{a-t}^{a} \rho(s) d s}=\frac{\xi_{2}(a)}{\xi_{2}(a-t)}, \\
\lambda(a) e^{-\int_{0}^{a} \lambda(s) d s} & =f_{1}(a), \quad \rho(a) e^{-\int_{0}^{a} \rho(s) d s}=f_{2}(a), \\
\lambda(a) e^{-\int_{a-t}^{a} \lambda(s) d s} & =\frac{f_{1}(a)}{\xi_{1}(a-t)}, \quad \rho(a) e^{-\int_{a-t}^{a} \rho(s) d s}=\frac{f_{2}(a)}{\xi_{2}(a-t)} .
\end{aligned}
$$

Substituting Eq. (5.17) and Eq. (5.18) into $p_{i}(t, a)$, we have

$$
p_{i}(t, a)= \begin{cases}\frac{p_{S}(t-a) \theta g^{\prime \prime}(\theta)}{g^{\prime}(\theta)} e^{-\int_{0}^{a} \mu d s+\int_{0}^{a} \int_{0}^{\infty} \lambda(u) p_{i}(t-a+s, u) d u d s} \xi_{1}(a) \xi_{2}(a) & \\ \int_{0}^{\infty} \lambda(a) p_{i}(t-a, a) d a & t>a \\ \frac{\epsilon \varphi(a-t)}{1-\epsilon} e^{-\int_{a-t}^{a} \mu d s+\int_{0}^{t} \int_{0}^{\infty} \lambda(u) p_{i}(s, u) d u d s \frac{\xi_{1}(a)}{\xi_{1}(a-t)} \frac{\xi_{2}(a)}{\xi_{2}(a-t)},} & t \leqslant a\end{cases}
$$

From Eq. (2.5), we have

$$
\begin{gathered}
p_{I}(t)=\int_{0}^{\infty} \lambda(a) p_{i}(t-a, a) d a \int_{0}^{t} \frac{p_{S}(t-a) \theta g^{\prime \prime}(\theta)}{g^{\prime}(\theta)} e^{-\int_{0}^{a} \mu d s+\int_{0}^{a} \int_{0}^{\infty} \lambda(u) p_{i}(t-a+s, u) d u d s} \xi_{1}(a) \xi_{2}(a) d a \\
\quad+\int_{t}^{\infty} \frac{\epsilon \varphi(a-t)}{1-\epsilon} e^{-\int_{a-t}^{a} \mu d s+\int_{0}^{t} \int_{0}^{\infty} \lambda(u) p_{i}(s, u) d u d s} \frac{\xi_{1}(a)}{\xi_{1}(a-t)} \frac{\xi_{2}(a)}{\xi_{2}(a-t)} d a .
\end{gathered}
$$

Applying Eq. (2.5) and the third equation of Eq. (2.6), we obtain

$$
\begin{aligned}
\dot{p}_{I}(t) & =\int_{0}^{\infty} \frac{\partial}{\partial t} p_{i}(t, a) d a \\
& =\int_{0}^{\infty}\left(-(\lambda(a)+\rho(a)+\mu) p_{i}(t, a)+p_{i}(t, a) \int_{0}^{\infty} \lambda(a) p_{i}(t, a) d a-\frac{\partial}{\partial a} p_{i}(t, a)\right) d a \\
& =\int_{0}^{\infty}\left[-(\lambda(a)+\rho(a)+\mu) p_{i}(t, a)\right] d a+\int_{0}^{\infty} \lambda(a) p_{i}(t, a) d a \int_{0}^{\infty} p_{i}(t, a) d a-p_{i}(t, \infty)+p_{i}(t, 0) \\
& =\int_{0}^{\infty}\left[-(\lambda(a)+\rho(a)+\mu) p_{i}(t, a)\right] d a+\int_{0}^{\infty} \lambda(a) p_{i}(t, a) d a p_{I}(t)-p_{i}(t, \infty)+p_{i}(t, 0)
\end{aligned}
$$

Again, along the characteristic lines $p_{\mathfrak{i}}(t, \infty)=p_{\mathfrak{i}}(0, \infty)=0$. Putting (5.19) into (5.20), we obtain

$$
\begin{aligned}
\dot{p}_{I}(t)= & \frac{p_{S}(t) \theta g^{\prime \prime}(\theta)}{g^{\prime}(\theta)} \int_{0}^{\infty} \lambda(a) p_{i}(t, a) d a-\mu p_{I}(t) \\
& -\left(1-p_{I}(t)\right) \int_{0}^{\infty} \lambda(a) p_{i}(t-a, a) d a \\
& \times \int_{0}^{t} \frac{p_{S}(t-a) \theta g^{\prime \prime}(\theta)}{g^{\prime}(\theta)} e^{-\int_{0}^{a} \mu d s+\int_{0}^{a} \int_{0}^{\infty} \lambda(u) p_{i}(t-a+s, u) d u d s} f_{1}(a) \xi_{2}(a) d a \\
& -\left(1-p_{I}(t)\right) \int_{t}^{\infty} \frac{\epsilon \varphi(a-t)}{1-\epsilon} e^{-\int_{a-t}^{a} \mu d s+\int_{0}^{t} \int_{0}^{\infty} \lambda(u) p_{i}(s, u) d u d s} \frac{f_{1}(a)}{\xi_{1}(a-t)} \frac{\xi_{2}(a)}{\xi_{2}(a-t)} d a \\
& -\int_{0}^{\infty} \lambda(a) p_{i}(t-a, a) d a \\
& \times \int_{0}^{t} \frac{p_{S}(t-a) \theta g^{\prime \prime}(\theta)}{g^{\prime}(\theta)} e^{-\int_{0}^{a} \mu d s+\int_{0}^{a} \int_{0}^{\infty} \lambda(u) p_{i}(t-a+s, u) d u d s} \xi_{1}(a) f_{2}(a) d a \\
& -\int_{t}^{\infty} \frac{\epsilon \varphi(a-t)}{1-\epsilon} e^{-\int_{a-t}^{a} \mu d s+\int_{0}^{t} \int_{0}^{\infty} \lambda(u) p_{i}(s, u) d u d s} \frac{\xi_{1}(a)}{\xi_{1}(a-t)} \frac{f_{2}(a)}{\xi_{2}(a-t)} d a .
\end{aligned}
$$

So we get the third equation of model (2.8). 